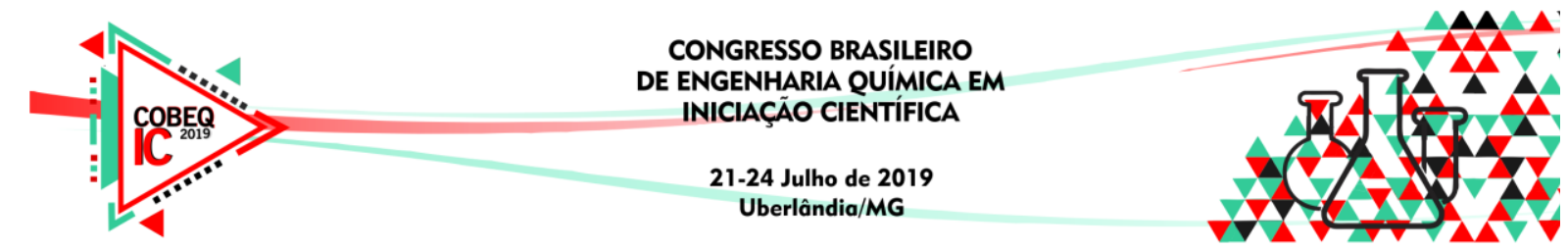

\title{
SIMULAÇÃO DE DIFERENTES CONDIÇÕES REACIONAIS NA POLIMERIZAÇÃO VIA RADICAIS LIVRES DE D- LIMONENO
}

\author{
F. M. COELHO ${ }^{1}$, R. P. VIEIRA ${ }^{1}$ \\ ${ }^{1}$ Universidade Estadual de Campinas, Faculdade de Engenharia Química \\ E-mail para contato: felipemouraoc8@gmail.com
}

\begin{abstract}
RESUMO - Atualmente, a esmagadora maioria dos plásticos utilizados no dia a dia provém de matriz não renovável e tóxica, sendo o petróleo a maior fonte. Dessa forma, considerável esforço vem sendo feito para se desenvolver alternativas às fontes atuais, sendo os plásticos verdes bons candidatos para tal. $\mathrm{O}$ limoneno, composto presente na casca dos frutos cítricos, possui potencial para polimerização, tendo em vista a sua dupla ligação residual. Porém, atualmente, se encontra bastante dificuldade para produzir esse polímero de modo que ele pudesse ser usado comercialmente, visto que as tentativas feitas resultaram em um polímero com baixa conversão de monômero e baixa massa molar média, devido às reações de transferência de cadeia. Assim, com o intuito de melhor analisar a cinética da polimerização do limoneno e as condições experimentais que a afetam, propôs se esse estudo. Inicialmente, foi desenvolvido um modelo matemático utilizando o método dos momentos para descrever a polimerização. Em seguida, a partir de dados encontrados na literatura para a conversão do limoneno, estimaram-se os parâmetros cinéticos envolvidos na reação. Os parâmetros característicos foram estimados em $k_{p}=165,9 \mathrm{M} \cdot \mathrm{s}^{-1}$ e $k_{f m}=4,021 \mathrm{M} \cdot \mathrm{s}^{-1}$. Concluiuse que o modelo não representou fidedignamente a realidade e são propostos novos modelos que considerem reações não elementares para dar continuidade a esse trabalho.
\end{abstract}

\section{INTRODUÇÃO}

Uma grande problemática no desenvolvimento de produtos poliméricos mundial atual se encontra no potencial de renovação de suas fontes e no seu impacto ambiental, visto que os combustíveis fósseis são suas principais matérias primas. Tendo em vista esse desafio, os plásticos de origem renovável e não tóxica despontam como potenciais substitutos aos atuais, havendo muitas áreas de pesquisa que focam no desenvolvimento daqueles (DUBÉ; SALEHPOUR, 2014).

Algumas das principais fontes de matéria prima para a síntese desses materiais "verdes" são a madeira, óleos essenciais e as fontes de amido. Uma das famílias de compostos orgânicos renováveis, que apresentam potencial para polimerização, são os terpenos, um dos principais constituintes de óleos essenciais de plantas cítricas. Esse potencial se dá uma vez que esses compostos apresentam uma ou mais unidades de isopreno em sua estrutura, 


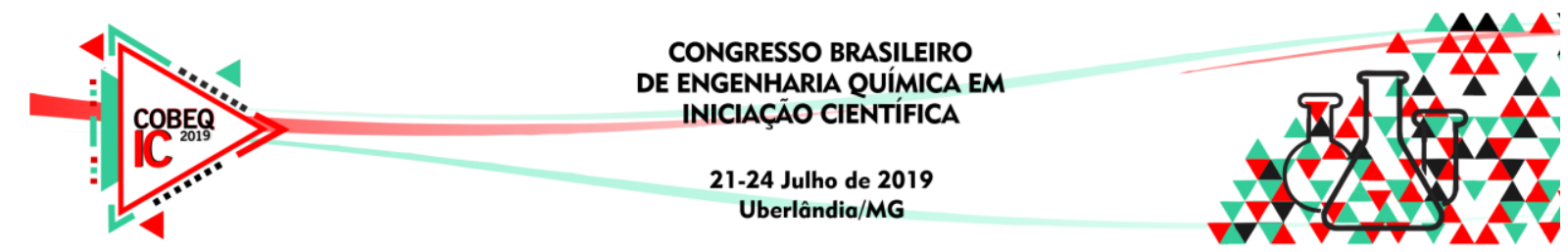

viabilizando, ao menos teoricamente, o processo de polimerização por adição (YAO; TANG, 2013).

Um dos compostos da família dos terpenos, que é objeto de estudo desse trabalho, é o limoneno. A capacidade produtiva mundial dessa substância a torna como potencial alternativa às fontes fósseis, visto que ele é extraído da casca da laranja - subproduto do processo de produção do suco. O Brasil, como maior produtor de suco de laranja do mundo, detêm assim um grande potencial em suas mãos. Atualmente, o país já é um grande produtor de óleos essenciais, porém praticamente nenhuma parte dessa produção é destinada à síntese de polímeros.

$\mathrm{Na}$ literatura existem estudos relacionados com a síntese do poli(limoneno), porém todos eles se deparam com o mesmo empecilho: a dificuldade de se obter uma conversão aceitável do monômero e um polímero com massa molar adequada. Roberts e Day (1950) polimerizaram D-limoneno em solução usando ácidos de Lewis e catalisadores de ZieglerNatta, obtendo produtos de massa molar em torno de 1000 g.mol ${ }^{-1}$ (menos de 10 unidades repetitivas). Recentemente, Singh e kamal (2012) realizaram a polimerização e caracterização do limoneno por via radicalar utilizando peróxido de benzó́la como iniciador em várias proporções. Os autores obtiveram conversão máxima de $12 \%$ e um polímero com temperatura de transição vítrea $(T g)$ em torno de $116{ }^{\circ} \mathrm{C}$ (um pouco superior a do poliestireno). Em reações de copolimerização radicalar com vários monômeros (estireno, metacrilato de metila e n-butil acrilato), as taxas de polimerização foram bastante reduzidas devido à presença de limoneno, e as massas molares dos copolímeros diminuíram com o aumento na proporção de limoneno (REN; TREVINO; DUBÉ, 2015).

A ocorrência dessa baixa conversão e massa molar média foi justificada pelos autores pela presença de reações de transferência de cadeia na estrutura do limoneno. A alta taxa de transferência de cadeia ocorre em virtude da presença dos hidrogênios alílicos, que também estão suscetíveis a gerarem radicais, porém estes são terminadores do crescimento polimérico.

Apesar do possível causador do problema ter sido apontado, há poucos estudos e pesquisas voltadas para fornecer possíveis soluções com o intuito de viabilizar a produção do poli(limoneno) em larga escala. Assim, através desse estudo propõem-se estimar parâmetros cinéticos da polimerização do limoneno, para que futuramente possa se estudar e determinar, através de simulações, condições operacionais que otimizam a conversão e massa molar do polímero final.

\section{METODOLOGIA}

\subsection{Modelagem}

A princípio almejava-se modelar uma reação de polimerização radicalar com transferência de cadeia puramente fenomenológica. Para tanto utilizou-se o método dos momentos (FERNANDES; LONA, 2004; MASTAN; ZHU, 2015) para modelar o processo de polimerização considerando as seguintes reações: iniciação, propagação, terminação com desproporcionamento, terminação com combinação e transferência de cadeia para o monômero. As equações obtidas para determinar os momentos para polímeros vivos $\left(Y_{i}\right)$ e 


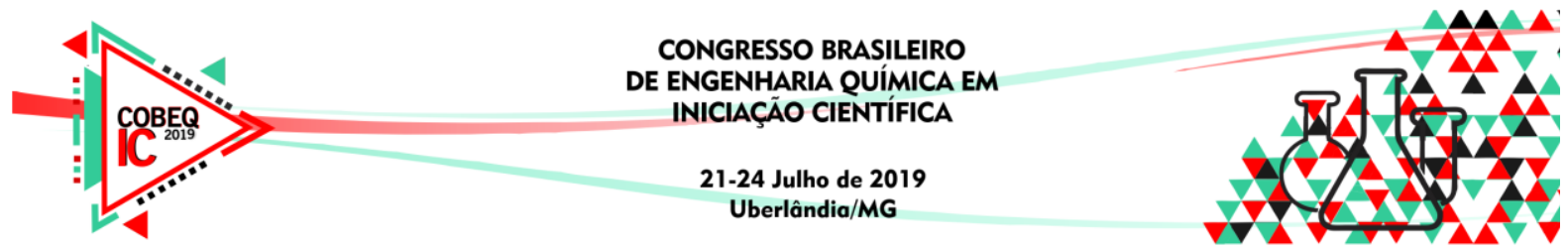

para os mortos $\left(Q_{i}\right)$ em função do tempo $(t)$, concentração do monômero $([M])$ e do iniciador $([I])$ e dos parâmetros cinéticos (iniciação $\left(k_{d}\right)$, propagação $\left(k_{p}\right)$, terminação por desproporcionamento $\left(k_{t d}\right)$, terminação por combinação $\left(k_{t c}\right)$ e transferência de cadeia para o monômero $\left(k_{f m}\right)$ ) estão elencadas abaixo:

$$
\begin{aligned}
& \frac{d Y_{0}}{d t}=2 f k_{d}[I]-\left(k_{t c}+k_{t d}\right) Y_{0}^{2} \\
& \frac{d Y_{1}}{d t}=k_{p}[M] Y_{0}+k_{f m}[M]\left(Y_{0}-Y_{1}\right)-\left(k_{t c}+k_{t d}\right) Y_{0} Y_{1} \\
& \frac{d Y_{2}}{d t}=k_{p}[M]\left(2 Y_{1}+Y_{0}\right)+k_{f m}[M]\left(Y_{0}-Y_{2}\right)-\left(k_{t c}+k_{t d}\right) Y_{0} Y_{2} \\
& \frac{d Q_{0}}{d t}=k_{f m}[M] Y_{0}+\left(\frac{k_{t c}}{2}+k_{t d}\right) Y_{0}{ }^{2} \\
& \frac{d Q_{1}}{d t}=k_{f m}[M] Y_{1}+\left(k_{t c}+k_{t d}\right) Y_{0} Y_{1} \\
& \frac{d Q_{2}}{d t}=k_{f m}[M] Y_{2}+\left(k_{t c}+k_{t d}\right) Y_{0} Y_{2}+k_{t c} Y_{1}^{2}
\end{aligned}
$$

Na etapa de iniciação também é inserido um termo relativo a eficiência do iniciador $(f)$ visto que nem todo iniciador que é ativado de fato inicia o processo de polimerização. A partir dessas equações pode-se estimar como varia a massa molar média numérica do polímero $(M n)$, a sua massa molar ponderal $(M w)$ e dispersidade $(D)$ através da massa molar do monômero (MM) (FERNANDES; LONA, 2004). Também é possível determinar a conversão do monômero $(X)$, relacionando a sua concentração atual $([M])$ com a sua concentração inicial $\left([M]_{0}\right)$ :

$$
\begin{aligned}
& M n=M M \frac{Q_{1}+Y_{1}}{Q_{0}+Y_{0}} \\
& M w=M M \frac{Q_{2}+Y_{2}}{Q_{1}+Y_{1}} \\
& D=\frac{M w}{M n}=\frac{\left(Q_{2}+Y_{2}\right)\left(Q_{0}+Y_{0}\right)}{\left(Q_{1}+Y_{1}\right)^{2}} \\
& X=\frac{[M]_{0}-[M]}{[M]_{0}}
\end{aligned}
$$

Como condição inicial do modelo diferencial, foi adotado que todos os momentos (vivos e mortos) eram iguais a zero e que as concentrações de monômero e iniciador eram iguais as concentrações iniciais respectivas.

\subsection{Programação}




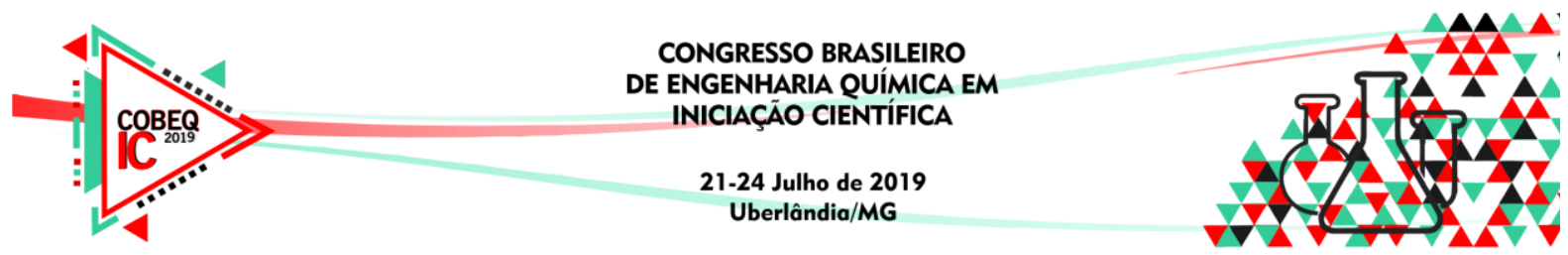

Para resolver o sistema de equações diferenciais ordinárias simultaneamente utilizouse do método numérico de Euler. Esse método foi escolhido pela sua facilidade e rápido tempo de resposta, comparado, por exemplo, ao método de Runge-Kutta de quarta ordem (a princípio esse método foi adotado e implementado no VBA do Excel, porém já nos primeiros testes ele se mostrou inviável dado o seu longo tempo de resposta, quando se chegava em uma resposta). Assim o método de Euler pode ser implementado nas próprias planilhas do Excel. Matematicamente, esse método é definido como:

$$
y_{n+1}=y_{n}+h f\left(x_{n}, y_{n}\right)
$$

Em que $h$ é o passo que deve ser selecionado. Nesse trabalho o passo é representado pelo tempo. $\mathrm{O}$ passo utilizado foi de $0,06 \mathrm{~s}$, pois esse foi o máximo possível que não gerou instabilidade no sistema determinar o maior passo que

\subsection{Estimativas de Parâmetros e Validação}

Para que se pudesse estimar os parâmetros cinéticos da polimerização do limoneno utilizaram-se os dados da conversão do monômero encontrados em estudos realizados por Singh e Kamal (2012). No estudo em questão, os autores objetivaram obter dados da cinética da reação de propagação do limoneno em poli(limoneno). Para tanto, reproduziram o ensaio de polimerização utilizando-se do BPO como iniciador a uma temperatura de $85^{\circ} \mathrm{C}$ sob uma atmosfera inerte de nitrogênio e utilizando xileno como solvente. Os dados obtidos para a conversão do limoneno ao longo do tempo para cada condição inicial de concentração se encontram transcritos na Tabela 1.

Tabela 1 - Conversão do monômero ao longo do tempo para diferentes concentrações iniciais de iniciador [I] e monômero [M]. Adaptado de Singh e Kamal (2012).

\begin{tabular}{|c|c|c|c|c|c|}
\cline { 3 - 6 } \multicolumn{2}{c|}{} & \multicolumn{4}{c|}{ Conversão do monômero $(\%)$} \\
\hline$[I]_{0} \mathrm{~mol} / \mathrm{L}$ & {$[M]_{0} \mathrm{~mol} / \mathrm{L}$} & 1 hora & 2 horas & 3 horas & 4 horas \\
\hline 0,0083 & 1,96 & 0,2 & 0,8 & 1,3 & 2,3 \\
\hline 0,0137 & 1,96 & 1 & 3 & 4,6 & 6,8 \\
\hline 0,0206 & 1,96 & 1,3 & 3,2 & 5,5 & 7,7 \\
\hline 0,0275 & 1,96 & 1,9 & 5,2 & 8,1 & 11,5 \\
\hline 0,0137 & 0,98 & 0,8 & 2,2 & 3,8 & 5,5 \\
\hline 0,0137 & 2,96 & 1,2 & 3,1 & 4,9 & 6,8 \\
\hline 0,0137 & 3,96 & 1,7 & 4,4 & 7,1 & 9,8 \\
\hline
\end{tabular}

Inicialmente, as constantes cinéticas de iniciação e de terminação (por combinação e terminação) foram estimadas. A constante de iniciação foi considerada igual a ordem de grandeza de iniciação do BPO para a temperatura reacional escolhida (aproximadamente $k_{d}=$ $10^{-5} \mathrm{~s}^{-1}$ ), com eficiência igual 0,6 e a de terminação igual a ordem de grandeza de terminação do poliestireno (aproximadamente $k_{t c}=k_{t d}=10^{8} \mathrm{M} \cdot \mathrm{s}^{-1}$ ). Assim, os demais parâmetros são característicos para a polimerização do limoneno. Para encontrá-los, utilizou-se a função de minimização do Solver do Excel (adotou-se o método GRG - gradientes reduzidos generalizados), comparando-se a conversão teórica (exibidas na Tabela 1) com a conversão calculada (determinada através da Equação 10), variando-se os parâmetros cinéticos $k_{p}$ e $k_{f m}$. 


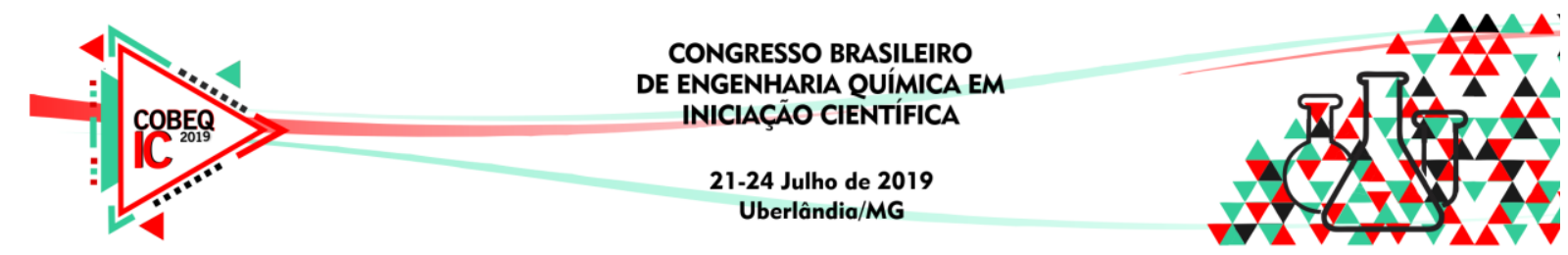

\section{RESULTADOS E DISCUSSÃO}

Abaixo, segue os gráficos obtidos do perfil da conversão do monômero ao longo do tempo para o modelo adotado para diversas concentrações iniciais de monômero e iniciador. Nos gráficos também foram plotados os pontos experimentais que constam na Tabela 1 para fins de comparação. Para facilitar a interpretação do efeito da concentração de monômero e de iniciador na conversão, o gráfico foi dividido em dois: em um manteve-se a concentração do monômero constante variando a do iniciador e no outro foi feito o inverso.

Figura 1 - Perfil da conversão do monômero utilizando a modelagem para: a) concentrações iniciais de iniciador, mantendo a concentração de monômero inicial fixa; b) concentrações iniciais de monômero, mantendo a concentração de iniciador inicial fixa.

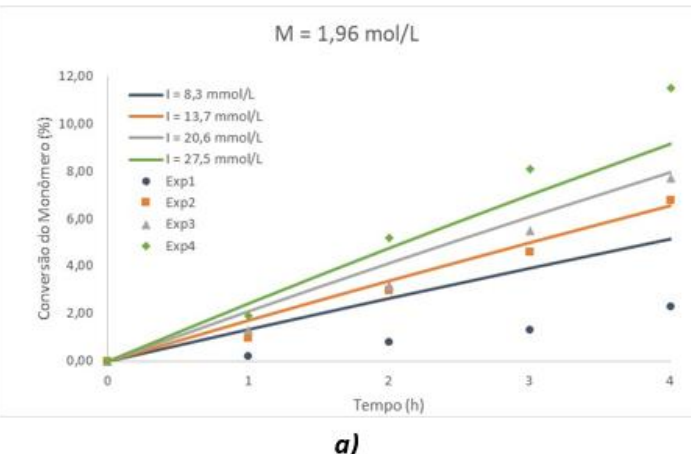

a)

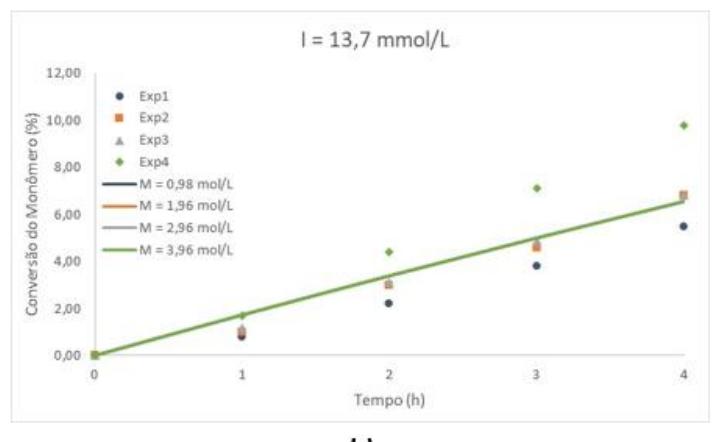

b)

Os parâmetros cinéticos estimados através da metodologia proposta encontrados para o modelo foram: $k_{p}=165,9 \mathrm{M} . \mathrm{s}^{-1}$ e $k_{f m}=4,021 \mathrm{M} \cdot \mathrm{s}^{-1}$.

Analisando-se a Figura 1 percebe-se que há uma discrepância entre a reta obtida e os pontos experimentais. A conversão do monômero para esse modelo, por exemplo, é independente da concentração inicial de monômero de acordo com o gráfico, como se pode observar na figura 1.b, o que não condiz com a realidade. Mostrando que a modelagem proposta não representa fielmente a realidade.

\section{CONCLUSÃO}

Com esse trabalho objetivou-se a determinação dos parâmetros cinéticos envolvidos na polimerização do limoneno. Considerando-se que o processo era composto apenas pelas reações de iniciação, propagação, terminação com desproporcionamento, terminação com combinação e transferência de cadeia para o monômero obteve-se um modelo não muito representativo com a realidade.

Assim, com o intuito de se alcançar resultados mais significativos, serão testados novos modelos. Nesses novos modelos, por exemplo, irá se adotar um sistema semi-empírico ao invés de um inteiramente fenomenológico. Dessa forma, considerará que as reações de propagação e transferência de cadeia são não elementares, o que resultará em novos parâmetros para serem determinados: a ordem de cada reação.

\section{REFERÊNCIAS}




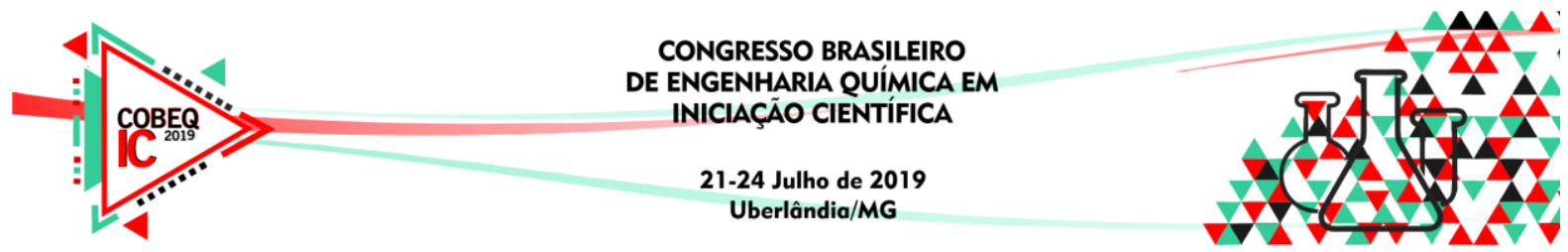

DUBÉ, M. A.; SALEHPOUR, S. Applying the Principles of Green Chemistry to Polymer Production Technology. Macromolecular Reaction Engineering, v. 8, n. 1, p. 7-28, 2014.

FERNANDES, Fabiano A. N.; LONA, Liliane M. F.. Introdução à modelagem de sistemas de polimerização. São Carlos: Editora Booklink, 2004. 140 p.

MASTAN, Erlita; ZHU, Shiping. Method of moments: A versatile tool for deterministic modeling of polymerization kinetics. European Polymer Journal, v. 68, p.139-160, jul. 2015. Elsevier BV. http://dx.doi.org/10.1016/j.eurpolymj.2015.04.018

REN, S.; TREVINO, E.; DUBÉ, M. A. Copolymerization of Limonene with n-Butyl Acrylate. Macromolecular Reaction Engineering, v. 9, n. 4, p. 339-349, 2015.

ROBERTS, W. J.; DAY, A. R. A Study of the Polymerization of $\alpha$ and $\beta$-Pinene with Friedel - Crafts Type Catalysts. Journal of the American Chemical Society, v. 72, n. 3, p. 1226-1230, 1950.

SINGH, Anamika; KAMAL, Meet. Synthesis and characterization of polylimonene: Polymer of an optically active terpene. Journal Of Applied Polymer Science, v. 125, n. 2, p.14561459, 14 jan. 2012. Wiley. http://dx.doi.org/10.1002/app.36250.

YAO, K.; TANG, C. Controlled polymerization of next-generation renewable monomers and beyond. Macromolecules, v. 46, n. 5, p. 1689-1712, 2013. 